\title{
Conservation Laws in Biochemical Reaction Networks
}

\author{
Adam Mahdi ${ }^{\mathrm{a}}$, Antoni Ferragut ${ }^{\mathrm{b}}$, Claudia Valls ${ }^{\mathrm{c}}$, Carsten Wiuf ${ }^{\mathrm{d}, *}$ \\ ${ }^{a}$ Institute of Biomedical Engineering, Department of Engineering Science, \\ University of Oxford, UK \\ ${ }^{b}$ Institut Universitari de Matemàtiques i Aplicacions de Castelló and Departament de \\ Matemàtiques, Universitat Jaume I, Castelló de la Plana, Spain \\ ${ }^{c}$ Departamento de Matemática, Instituto Superior Técnico, Lisboa, Portugal \\ ${ }^{d}$ Department of Mathematical Sciences, University of Copenhagen, Denmark
}

\begin{abstract}
We study the existence of linear and non-linear conservation laws in biochemical reaction networks with mass-action kinetics. It is straightforward to compute the linear conservation laws as they are related to the left null-space of the stoichiometry matrix. The non-linear conservation laws are difficult to identify and have rarely been considered in the context of mass-action reaction networks. Here, using Darboux theory of integrability we provide necessary structural (i.e. parameter independent) conditions on a reaction network to guarantee the existence of non-linear conservation laws of certain type. We give necessary and sufficient structural conditions for the existence of exponential factors with linear exponents and univariate linear Darboux polynomials. This allows us to conclude that a non-linear first integrals (similar to Lotka-Volterra system) only exists under the same structural condition. We finally show that the existence of such a first integral generally implies that the system is persistent and has stable steady states. We illustrate our results by examples.
\end{abstract}

Keywords: Darboux polynomials; dynamical systems; mass-action; non-linear conservation law; persistence; Lotka-Volterra

${ }^{*}$ Corresponding author 


\section{Introduction}

Typical models of biochemical reaction networks are systems of polynomial ordinary differential equations (ODEs). Generally, these systems cannot be solved explicitly and, except some special cases [8, 25], little is know about their

5 solution structure. In many applications it is important to know the qualitative aspects of the reaction network; whether the solutions are periodic, attracted towards some steady states, or persistent. An approach is to look for first integrals (or conservation laws), quantities depending on the state variables that are conserved over time. In connection with reaction networks, first integrals have been used to demonstrate various phenomena including oscillatory behaviour [25], chaos 24] and Turing instability 22].

Here our aim is twofold. Firstly, we are interested in finding sufficient and/or necessary structural conditions - that is, conditions that are independent of the parameters - for the existence of non-linear first integrals. Secondly, we 15 study the relationship between the existence of non-linear first integrals and the qualitative aspects of the reaction network. We make use of the Darboux theory of integrability [5, 6, which is a powerful tool for determining the non-linear first integrals [18, 16].

Regarding our first aim, we give the necessary and sufficient structural condition for the existence of exponential factors of the form $E(x)=\exp \left(\sum_{i} \omega_{i} x_{i}\right)$, $\omega_{i} \in \mathbb{R}$. Further, we characterize reaction networks that admit a linear Darboux polynomials (see Definition 12 of the form $F(x)=x_{i}$ from which we deduce the existence of non-nonlinear first integrals. Regarding our second aim, we show that the existence of a first integral (of a certain form) for a conservative system

25 implies that it is persistent and has a unique non-attracting, but stable, steady state in each stoichiometric compatibility class.

Although the use of linear conservation laws in reaction network theory is common, the non-linear first integrals have rarely been considered, with the exception of some special cases [15, 1, 17, 11, 12, 20. Quadratic first integrals have been characterized in [20]. Ideally, one would like to give structural condi- 
tions for the existence of first integrals (of certain forms) - irrespectively of the reaction rate constants. Our results can be seen as a first step in this direction, which is analogous to the deficiency zero and one theorems that give structural conditions for the existence of steady states [8] and the persistence criteria [2].

In general, it is difficult to prove the existence or non-existence of Darboux first integrals, which often depends on the reaction rate constants in complicated ways [11, 12, 17, 10. We hope that this paper would inspire further work to characterize the existence and form of non-linear first integrals for reaction networks. As our results show, non-linear first integrals might be useful to study qualitative aspects of the dynamics.

\section{Reaction networks with mass-action kinetics}

Here we define a reaction network and show how to construct a system of polynomial ODEs describing the evolution of the species concentrations.

\subsection{Reaction networks}

Denote by $\mathbb{N}$ the set of non-negative integers and by $\mathbb{R}_{>0}\left(\mathbb{R}_{\geq 0}\right)$ the set of positive (non-negative) real numbers. Let $e_{i}$ be the $i$ th unit vector of $\mathbb{R}^{n}$. For $x=\left(x_{1}, \ldots, x_{n}\right) \in \mathbb{R}_{>0}^{n}$ and $\alpha=\left(\alpha_{1}, \ldots, \alpha_{n}\right) \in \mathbb{R}^{n}$, let $x^{\alpha}=\prod_{i=1}^{n} x_{i}^{\alpha_{i}}$. If $\alpha_{i} \geq 0$ for all $i=1, \ldots, n$, we allow $x \in \mathbb{R}_{\geq 0}^{n}$. In the following, $\Omega$ denotes an open set in $\mathbb{R}^{n}$.

50 Definition 1 (Reaction network). A reaction network is a triplet $(\mathcal{S}, \mathcal{C}, \mathcal{R})$ :

(a) $\mathcal{S}=\left\{A_{1}, \ldots, A_{n}\right\}$ has $n$ elements, the species.

(b) $\mathcal{C}$ has $p$ elements, the complexes, being linear combinations of species, $y=\alpha_{1} A_{1}+\ldots+\alpha_{n} A_{n} \in \mathcal{C}$, with $\alpha_{i} \in \mathbb{N}, i=1, \ldots, n$.

(c) $\mathcal{R}$ has $m$ elements, the reactions, and each reaction $r \in \mathcal{R}$ is an ordered pair of distinct complexes, written $y \rightarrow y^{\prime}$. Therefore $\mathcal{R} \subseteq \mathcal{C} \times \mathcal{C}$. 
In the following we assume that the sets $\mathcal{S}, \mathcal{C}$ and $\mathcal{R}$ are ordered. It is common to assume that $\mathcal{C}$ only contains complexes which are part of reactions and $\mathcal{S}$ only contains species which are part of complexes. In that case $\mathcal{S}$ and $\mathcal{C}$ are determined by $\mathcal{R}$. For later use we define the set of reactant complexes ${ }_{60} \mathcal{C}^{\prime}=\left\{y \in \mathcal{C} \mid y \rightarrow y^{\prime} \in \mathcal{R}\right\} \subseteq \mathcal{C}$, that is, the complexes on the left hand side of the reactions.

Example 1 (Volpert's network). Consider the following reaction network [25]:

$$
A_{1}+A_{2} \longrightarrow 2 A_{2}, \quad A_{2}+A_{3} \longrightarrow 2 A_{3}, \quad A_{3}+A_{1} \longrightarrow 2 A_{1}
$$

It has six complexes $\mathcal{C}=\left\{A_{1}+A_{2}, 2 A_{2}, A_{2}+A_{3}, 2 A_{3}, A_{3}+A_{1}, 2 A_{1}\right\}$ and three species $\mathcal{S}=\left\{A_{1}, A_{2}, A_{3}\right\}$.

A reaction network $(\mathcal{S}, \mathcal{C}, \mathcal{R})$ has a natural representation in terms of a (directed) graph, as in Example 1. where the vertices are the complexes and the (directed) edges between vertices are the reactions. The connected components of the undirected graph are called linkage classes and the strongly connected components of the directed graph are called terminal linkage classes. In Example 1 there are three linkage classes (e.g., $A_{1}+A_{2} \rightarrow 2 A_{2}$ ) and three terminal linkage classes (e.g., $\left.2 A_{2}\right)$.

\subsection{Stoichiometric subspace and compatibility class}

Consider a reaction network $(\mathcal{S}, \mathcal{C}, \mathcal{R})$. Identify each species $A_{i}$ with the $i$-th unit vector in $\mathbb{N}^{n}$, that is, the vector with one in the $i$-th entry and zero elsewhere. Thus, a complex $y=\alpha_{1} A_{1}+\ldots+\alpha_{n} A_{n}$ is identified with the element ${ }_{75}\left(\alpha_{1}, \ldots, \alpha_{n}\right)$ of $\mathbb{R}^{n}$.

For the $j$-th reaction $y_{j} \rightarrow y_{j}^{\prime}$, we let $\gamma_{j}=y_{j}^{\prime}-y_{j} \in \mathbb{R}^{m}$ be the net production of species in the reaction. The vector $\gamma_{j}$ is referred to as the $j$-th reaction vector.

Definition 2 (Stoichiometry matrix). The stoichiometry matrix $\Gamma$ is defined as the $n \times m$ matrix whose columns are the reaction vectors $\Gamma=\left(\gamma_{1}, \gamma_{2}, \ldots, \gamma_{m}\right)$.

so Definition 3 (Stoichiometric subspace). The stoichiometric subspace is the vector subspace $S \subseteq \mathbb{R}^{n}$ defined as $S=\operatorname{span}\left\{\gamma_{1}, \ldots, \gamma_{m}\right\}$. 
Definition 4 (Stoichiometric compatibility class). The stoichiometric compatibility classes are the affine subspaces of $\mathbb{R}^{n}$ defined by $(x+S) \cap \mathbb{R}_{\geq 0}^{n}$, where $x \in \mathbb{R}_{\geq 0}^{n}$.

Let $(\mathcal{S}, \mathcal{C}, \mathcal{R})$ be a reaction network. Denote by $x_{i}$ the concentration of the species $A_{i}$ and by $x(t)=\left(x_{1}(t), \ldots, x_{n}(t)\right)$ the vector of species concentrations. Before we show how to describe the time evolution of the vector of concentrations, we need some preliminary definitions and notation.

A rate function for a reaction $y_{j} \rightarrow y_{j}^{\prime}$, for $j=1, \ldots, m$, is some function $v_{j}(x): \mathbb{R}_{\geq 0}^{n} \rightarrow \mathbb{R}_{\geq 0}$ that describes the instantaneous change in the species composition $x=\left(x_{1}, \ldots, x_{n}\right)$ due to this reaction.

A kinetics $\mathcal{V}$ is an assignment to each reaction $y_{j} \rightarrow y_{j}^{\prime}, j=1, \ldots, m$, of a rate function $v_{j}(x): \mathbb{R}_{\geq 0}^{n} \rightarrow \mathbb{R}_{\geq 0}$. We can think of $\mathcal{V}$ as a set of rate functions indexed by the elements of the reaction set, that is $\mathcal{V}=\left\{v_{j}(x): j=1, \ldots, m\right\}$. Let $v(x)=\left(v_{1}(x), \ldots, v_{m}(x)\right)$ be the vector of rate functions. We will also refer to the function $v(x): \mathbb{R}_{\geq 0}^{n} \rightarrow \mathbb{R}_{\geq 0}^{m}$ as the kinetics.

Definition 5 (ODE system of a reaction network). Let $(\mathcal{S}, \mathcal{C}, \mathcal{R})$ be a reaction network. The evolution of the concentrations $x(t)=\left(x_{1}(t), \ldots, x_{n}(t)\right)$ under the kinetics $\mathcal{V}$ is given by the ODE system

$$
\frac{d x}{d t}=\sum_{j=1}^{m} v_{j}(x) \gamma_{j}=\Gamma v(x), \quad \text { and } \quad x(0)=x_{0} \in \mathbb{R}_{\geq 0}^{n},
$$

where $v(x)=\left(v_{1}(x), \ldots, v_{m}(x)\right)$ is the vector of the rate functions.

It follows from (2) that the evolution of the species concentrations is confined to the stoichiometric subspace

$$
x(t) \in\left(x_{0}+S\right) \cap \mathbb{R}_{\geq 0}^{n}, \quad \text { where } \quad x(0)=x_{0} .
$$

Non-negativity of the solutions follows from the assumption that the rate functions are non-negative, in particular at the boundary of $\mathbb{R}_{\geq 0}^{n}[21$. 
We are particularly interested in mass-action kinetics.

Definition 6 (Mass-action kinetics). A kinetics is called mass-action if the rate functions take the form

$$
v_{\kappa, j}(x)=k_{j} \prod_{i=1}^{n} x_{i}^{\alpha_{j i}}=k_{j} x^{y_{j}}
$$

where $y_{j}=\left(\alpha_{j 1}, \ldots, \alpha_{j n}\right)$, and $k_{j}>0$ is a positive reaction rate constant. We denote the vector of reaction rate constants by $\kappa=\left(k_{1}, \ldots, k_{m}\right)$.

Remark 7. Note that the total degree of a mass-action rate function $v_{\kappa, j}(x)$ ${ }_{105}$ is $\operatorname{deg}\left(v_{\kappa, j}(x)\right)=\sum_{i=1}^{n} \alpha_{j i}$.

Example 2 (Volpert's network, part 2). Consider the reaction network given in (1) with mass-action kinetics. The corresponding system of ODEs is given by

$$
\frac{d x}{d t}=\Gamma v_{\kappa}(x)=\left(\begin{array}{l}
k_{3} x_{1} x_{3}-k_{1} x_{1} x_{2} \\
k_{1} x_{1} x_{2}-k_{2} x_{2} x_{3} \\
k_{2} x_{2} x_{3}-k_{3} x_{1} x_{3}
\end{array}\right)
$$

where

$$
\Gamma=\left(\begin{array}{rrr}
-1 & 0 & 1 \\
1 & -1 & 0 \\
0 & 1 & -1
\end{array}\right) \quad \text { and } \quad v_{\kappa}(x)=\left(\begin{array}{l}
k_{1} x_{1} x_{2} \\
k_{2} x_{2} x_{3} \\
k_{3} x_{1} x_{3}
\end{array}\right)
$$

\subsection{Linear conservation laws}

Proposition 8. Let (2) be an ODE system for a reaction network. For any row vector $\omega=\left(\omega_{1}, \omega_{2}, \ldots, \omega_{n}\right)$ such that $\omega \Gamma=0$, the quantity

$$
H=\sum_{i=1}^{n} \omega_{i} x_{i}
$$

is a linear conservation law. Moreover, there are at least $s=n-\operatorname{rank}(\Gamma)$ independent linear conservation laws. 
Proof. We have

$$
\frac{d H}{d t}=\omega \frac{d x}{d t}=\omega \Gamma v(x)=0 .
$$

The left null-space of $\Gamma$ has dimension $s$, hence there must be at least $s$ independent linear conservation laws.

Example 3. Consider the reaction network

$$
A_{1} \stackrel{k_{1}}{\longrightarrow} A_{2}, \quad A_{1} \stackrel{k_{2}}{\longrightarrow} A_{3} .
$$

In this case, $s=1$ as $H_{1}=x_{1}+x_{2}+x_{3}$ is a conserved quantity corresponding to the vector $\omega^{1}=(1,1,1)$. It is easy to see that $H_{2}=k_{2} x_{2}-k_{1} x_{3}$ is another linear conservation law for all $\kappa=\left(k_{1}, k_{2}\right) \in \mathbb{R}_{>0}^{2}$, although $\omega^{2}=\left(0, k_{2},-k_{1}\right)$ is not in the null-space of $\Gamma$. The two vectors $\omega^{1}$ and $\omega^{2}$ are linearly independent for all reaction rate constants.

Theorem 9 (see [9]). Let $S_{\kappa}=\operatorname{span}\left(\Gamma v_{\kappa}\left(\mathbb{R}_{\geq 0}^{n}\right)\right) \subseteq S$ for a reaction network with mass-action kinetics, as in (3). If the number of linkage classes equals the number of terminal linkage classes, then $S_{\kappa}=S$ and the number of independent linear conservation laws is $s=n-\operatorname{rank}(\Gamma)$.

Example 4. In the reaction network (5) of Example 3 , there is one linkage class but two terminal linkage classes $\left(A_{1}\right.$ and $\left.A_{2}\right)$, hence the proposition does not apply. As a second example, consider the reaction network

$$
A_{1} \stackrel{k_{1}}{\longrightarrow} 2 A_{1}, \quad A_{1} \stackrel{k_{2}}{\longrightarrow} 0 .
$$

120 in the left null-space of $\Gamma$, that is, $\omega$ is not of the form in Proposition 8 . Indeed $s=0$. If $k_{1} \neq k_{2}$ then there are no linear conservation laws. Indeed, $S_{(k, k)}=\mathbb{R}$, while $S_{\left(k_{1}, k_{2}\right)}=\{0\}$ for $k_{1} \neq k_{2}$.

Example 2 (see also Example 1) has three linkage classes and three terminal 125

$$
H=x_{1}+x_{2}+x_{3} \text {. }
$$


Theorem 9 gives a structural condition that guarantees the existence of precisely $s$ linear independent conservation laws, which can be chosen independently of $\kappa$. In general, the linear conservation laws depend on the kinetics. Under mass-action kinetics this dependence can be made precise, as alluded to in the examples above. For a given $\kappa$, let $\Gamma_{\kappa}$ be the $m \times p^{\prime}$-matrix ( $p^{\prime}$ being the number of reactant complexes in $\mathcal{C}$ ) with column corresponding to the reactant complex $y \in \mathcal{C}^{\prime}$ given by

$$
\sum_{j=1, y_{j}=y}^{m} k_{j}\left(y_{j}^{\prime}-y_{j}\right),
$$

such that $\dot{x}=\Gamma v_{\kappa}(x)=\sum_{y \in \mathcal{C}^{\prime}} \sum_{j=1, y_{j}=y}^{m} k_{j}\left(y_{j}^{\prime}-y_{j}\right) x^{y}$. Then, the linear conservation laws are of the form $H=\sum_{i=1}^{n} \omega_{i} x_{i}$, where $\omega=\left(\omega_{1}, \ldots, \omega_{n}\right)$ is in the left null-space of $\Gamma_{\kappa}$ [9], and there are $s_{\kappa}=n-\operatorname{rank}\left(\Gamma_{\kappa}\right)$ such independent conservation laws. For example in the reaction network (6), we have

$$
\Gamma=(1-1) \quad \text { and } \quad \Gamma_{\kappa}=\left(k_{1}-k_{2}\right)
$$

( $\Gamma$ is $1 \times 2$-dimensional and $\Gamma_{\kappa}$ is $1 \times 1$-dimensional), resulting in the conservation laws previously stated.

\section{Non-linear conservation laws via Darboux theory}

The theory developed by Darboux [5, 6] is one of the most useful tools for identifying the first integrals of polynomial differential equations. Here we review the main elements of the Darboux theory and show how it can be applied to search for non-linear conservation laws of the reaction networks with massaction kinetics.

We start by considering an $n$-dimensional polynomial differential system

$$
\frac{d x_{1}}{d t}=P_{1}(x), \ldots, \frac{d x_{n}}{d t}=P_{n}(x),
$$

where $x=\left(x_{1}, \ldots, x_{n}\right) \in \mathbb{R}^{n}$ and $P_{i}(x) \in \mathbb{R}[x]$ are polynomials in $x$. Whenever convenient we omit the argument $x$ in $P_{i}=P_{i}(x)$ and other functions of $x$. 
For any system of differential equations (8) we define a derivation

$$
\mathcal{D}:=P_{1} \frac{\partial}{\partial x_{1}}+\ldots+P_{n} \frac{\partial}{\partial x_{n}} .
$$

The derivation evaluated at a $\mathcal{C}^{1}$-function $H: \Omega \rightarrow \mathbb{R}$, where $\Omega$ can be taken to be $\mathbb{R}_{>0}^{n}$, is denoted by

$$
\mathcal{D}(H):=P_{1} \frac{\partial H}{\partial x_{1}}+\cdots+P_{n} \frac{\partial H}{\partial x_{n}} .
$$

The degree of system (8), or of the derivation (9), is defined as

$$
d=\max \left(\operatorname{deg}\left(P_{1}\right), \ldots, \operatorname{deg}\left(P_{n}\right)\right),
$$

where $\operatorname{deg}(P)$ denotes the degree of polynomial $P$.

Remark 10. Consider a reaction network with mass-action kinetics and assume that none of the monomials in the rate functions vanish in the expression of $P_{i}$, $i=1, \ldots, n$. According to Remark 7 the degree of the system is

$$
d^{\prime}=\max \left(\sum_{i=1}^{n} \alpha_{j, i} \mid y_{j}=\left(\alpha_{j 1}, \ldots, \alpha_{j n}\right), y_{j} \rightarrow y_{j}^{\prime}\right) \text {. }
$$

Even if the hypotheses of Theorem 9 hold, it can still be that some monomials vanish. This is the case for the network $2 A \stackrel{k_{1}}{\longrightarrow} 3 A, 2 A \stackrel{k_{2}}{\longrightarrow} A, A \stackrel{k_{3}}{\longrightarrow}$ $B, B \stackrel{k_{4}}{\longrightarrow} 2 A$, which has one linkage class and one terminal linkage class. If $k_{1}=k_{2}$, then $d=1$, and if $k_{1} \neq k_{2}$, then $d=2$, but we always have $d^{\prime}=2$.

We refer to $d^{\prime}$ as the structural degree of the system. In general $d \leq d^{\prime}$. There always exists reaction rate constants $\kappa$ such that $d=d^{\prime}$.

Definition 11 (First integral). We say that a $\mathcal{C}^{1}$-function $H: \Omega \rightarrow \mathbb{R}$, where ${ }_{145} \Omega \subseteq \mathbb{R}^{n}$, is a first integral of system (8), or of the derivation $\mathcal{D}$, if $\mathcal{D}(H)=0$, and $H$ is not locally constant on any positive Lebesgue measure subset of $\Omega$.

Definition 12 (Darboux polynomial). A Darboux polynomial of system (8) is 
a polynomial $F \in \mathbb{C}[x]$, such that

$$
\mathcal{D}(F):=P_{1} \frac{\partial F}{\partial x_{1}}+\cdots+P_{n} \frac{\partial F}{\partial x_{n}}=K F
$$

for some cofactor $K \in \mathbb{C}[x]$.

Remark 13. A polynomial first integral is also a Darboux polynomial with cofactor $K=0$. If the degree of the derivation $(9)$ is $d$, then the degree of any cofactor in 11] is bounded by $d-1$.

Definition 14 (Exponential factor). An exponential factor of system (8) is a function $E=\exp (G / F)$, with $F, G \in \mathbb{C}[x]$, such that

$$
\mathcal{D}(E):=P_{1} \frac{\partial E}{\partial x_{1}}+\cdots+P_{n} \frac{\partial E}{\partial x_{n}}=L E,
$$

for some cofactor $L \in \mathbb{C}[x]$, where the degree of $L$ is lower than the degree of $\mathcal{D}$. A linear exponential factor is an exponential factor such that $F \equiv 1$ and $G$ is a linear function of $x$.

Remark 15. Note that $F$ in the expression $E=\exp (G / F)$ is a Darboux polynomial. Moreover it can be easily shown that $\mathcal{D}(G)=K_{F} G+L F$, where $K_{F}$ is the cofactor of $F$. If $E$ is a linear exponential factor, then $\mathcal{D}(G)=L$ as $F \equiv 1$ and $K_{F}=0$.

The Darboux theory of integrability relates the number of Darboux polynomials and exponential factors with the existence of a Darboux first integral [26]. A Darboux function is a product of complex powers of Darboux polynomials and exponential factors. Note that a rational function (a ratio of two polynomials) is a special case of a Darboux function. A Darboux first integral is a Darboux function that is a first integral according to Definition 11 .

Theorem 16. Assume that a derivation $\mathcal{D}$ of degree $d$ admits $r$ Darboux polynomials $F_{i}, i=1, \ldots, r$, and $s$ exponential factors $E_{j}, j=1, \ldots, s$. Let $N=\left(\begin{array}{c}n+d-1 \\ n\end{array}\right)$. Then the following statements hold. 
(a) If $r+s \geq N+1$, then the derivation $\mathcal{D}$ admits a Darboux first integral.

(b) If $r+s \geq N+n$, then the derivation $\mathcal{D}$ admits a rational fist integral.

Theorem 16(a) is due to Darboux [6, 5], whereas Theorem 16,b) is attributed 170 to Jouanolou [14]. Theorem 16 reduces the task of identifying the first integrals of a differential system to computing its Darboux polynomials.

As an illustration of the bound in Theorem 16(a) consider the case $n=2$, that is, a system in two variables. As noted earlier, for Darboux polynomials $F_{i}$ with cofactors $K_{i}$ and exponential factors $E_{j}$ with cofactors $L_{j}$, we have

$$
\max \left\{\operatorname{deg} K_{1}, \ldots, \operatorname{deg} K_{r}, \operatorname{deg} L_{1}, \ldots, \operatorname{deg} L_{s}\right\} \leq d-1
$$

Since the number of linearly independent polynomials in two variables of degree at most $d-1$ is $\left(\begin{array}{c}d+1 \\ 2\end{array}\right)$, there must be a linear combination of cofactors summing to zero, whenever $r+s$ is strictly bigger than $\left(\begin{array}{c}d+1 \\ 2\end{array}\right)$. Hence Theorem 16 applies.

Theorem 17. Assume that the derivation $\mathcal{D}$ admits $r$ Darboux polynomials $F_{i}$ with respective cofactors $K_{i}, i=1, \ldots, r$, and $s$ exponential factors $E_{j}$ with respective cofactors $L_{j}, j=1, \ldots, s$. If there exist $\lambda_{1}, \ldots, \lambda_{r}, \mu_{1}, \ldots, \mu_{s}$ in $\mathbb{C}$, such that not all are zero and

$$
\sum_{i=1}^{r} \lambda_{i} K_{i}+\sum_{j=1}^{s} \mu_{j} L_{j}=0
$$

then the Darboux function

$$
\Phi(x)=F_{1}^{\lambda_{1}} \cdots F_{r}^{\lambda_{r}} E_{1}^{\mu_{1}} \cdots E_{s}^{\mu_{s}}
$$

175 is a first integral of (8), provided $\Phi$ is not locally constant on any positive Lebesgue measure subset of its domain of definition.

Proof. We first note that if $\Phi(x)$ is a first integral, then the composition $F(\Phi(x))$ is another first integral, where $F$ is any $\mathcal{C}^{1}$-function. Now we prove that $\log \Phi$ 
is a first integral. We have

$$
\begin{aligned}
\mathcal{D}(\log \Phi) & =\sum_{i=1}^{r} \lambda_{i} \mathcal{D}\left(\log F_{i}\right)+\sum_{j=1}^{s} \mu_{j} \mathcal{D}\left(\log E_{j}\right) \\
& =\sum_{i=1}^{r} \lambda_{i} \frac{\mathcal{D}\left(F_{i}\right)}{F_{i}}+\sum_{j=1}^{s} \mu_{j} \frac{\mathcal{D}\left(E_{j}\right)}{E_{j}}=\sum_{i=1}^{r} \lambda_{i} K_{i}+\sum_{j=1}^{s} \mu_{j} L_{j} .
\end{aligned}
$$

The theorem follows from 12 .

\section{Linear exponential factors}

Now, we characterize the linear exponential factors with non-zero cofactors

Proposition 18. Let (2) be an n-dimensional ODE system for a reaction network with mass-action kinetics, $v_{\kappa}(x)=\left(v_{\kappa, 1}(x), \ldots, v_{\kappa, m}(x)\right)$, as in (3), and let $d^{\prime}$ be the structural degree of the system, as in 100 . Further, let $\Gamma=\left(\Gamma^{1}, \Gamma^{2}\right)$ be the stoichiometric matrix with the columns potentially reordered such that $\Gamma^{1}$ consists of the columns for which $\operatorname{deg}\left(v_{\kappa, j}\right)(x)=d^{\prime}, j=1, \ldots, m$, and $\Gamma^{2}$ consists of the remaining columns. Then, for any row vector $\omega=\left(\omega_{1}, \ldots, \omega_{n}\right)$ such that $\omega \Gamma^{1}=0$, the quantity

$$
E=\exp (G), \quad \text { with } \quad G=\sum_{j=1}^{m} \omega_{j} x_{j}
$$

is a linear exponential factor with cofactor $L=\omega \Gamma^{2} v_{\kappa}^{2}(x)$. Moreover, there are at least $s^{\prime}=\operatorname{rank}(\Gamma)-\operatorname{rank}\left(\Gamma^{1}\right)$ independent linear exponential factors with non-zero cofactors. 
Proof. Let $\Gamma^{1}$ be $n \times m_{1}$-dimensional and $\Gamma^{2}$ be $n \times m_{2}$-dimensional, such that $m_{1}+m_{2}=m$. Further, let $v_{\kappa}(x)=\left(v_{\kappa}^{1}(x), v_{\kappa}^{2}(x)\right)$ be the vector of rate functions potentially reordered to reflect the reordering of $\Gamma=\left(\Gamma^{1}, \Gamma^{2}\right)$, that is, $\operatorname{deg}\left(v_{\kappa, j}^{1}(x)\right)=d^{\prime}, j=1, \ldots, m_{1}$, and $\operatorname{deg}\left(v_{\kappa, j}^{2}(x)\right)<d^{\prime}, j=m_{1}+1, \ldots, m$. For $E=\exp (G)$ with $G=\sum_{i=1}^{n} \omega_{i} x_{i}$ as in the statement of the proposition, we have $\mathcal{D}(E)=E \mathcal{D}(G)$ and

$$
\mathcal{D}(G)=\sum_{i=1}^{n} P_{i} \frac{\partial G}{\partial x_{i}}=\omega \Gamma v_{\kappa}(x)=\omega \Gamma^{2} v_{\kappa}^{2}(x)
$$

As $\operatorname{deg}\left(v_{\kappa, j}^{2}(x)\right)<d^{\prime}, j=m_{1}+1, \ldots, m$, then $\operatorname{deg}(\mathcal{D}(G))<d^{\prime}$, where $d^{\prime}$ is the degree of the derivation. Hence $E$ is a linear exponential factor with the desired cofactor.

There are at least $n-\operatorname{rank}\left(\Gamma^{1}\right)$ linearly independent exponential factors of this kind. However, not all of these have non-zero cofactor. Any linear conservation law gives rise to a linear exponential factor with zero cofactor. Hence, discounting the linear conservation laws for which $\omega \Gamma=0$ yields that there are at least $\left(n-\operatorname{rank}\left(\Gamma^{1}\right)\right)-(n-\operatorname{rank}(\Gamma))=\operatorname{rank}(\Gamma)-\operatorname{rank}\left(\Gamma^{1}\right)$ independent linear exponential factors with non-zero cofactor.

If $\operatorname{rank}(\Gamma)=\operatorname{rank}\left(\Gamma^{1}\right)$ (which is the case if all reactions have the same order, that is, $\operatorname{deg}\left(v_{\kappa, j}(x)\right)=\sum_{i=1}^{n} \alpha_{j i}=d^{\prime}$ for all $\left.j=1, \ldots, m\right)$, then the proposition does not give any non-trivial linear exponential factors.

Remark 19. We can also state a parameter dependent version of Proposition 18. Let $d_{\kappa}$ be the degree of the system for a given $\kappa, \Gamma_{\kappa}$ be defined as in (7) and $\Gamma_{\kappa}=\left(\Gamma_{\kappa}^{1}, \Gamma_{\kappa}^{2}\right)$ be a partition of $\Gamma_{\kappa}$ defined in Proposition 18 . Then Proposition 18 holds with $d^{\prime}$ replaced by $d_{\kappa}$ and $\Gamma=\left(\Gamma^{1}, \Gamma^{2}\right)$ replaced by $\Gamma_{\kappa}=\left(\Gamma_{\kappa}^{1}, \Gamma_{\kappa}^{2}\right)$. ${ }_{205}$ Consider the reaction network defined by the reactions $\mathcal{R}^{1}=\left\{y_{j} \rightarrow y_{j}^{\prime} \mid j=\right.$ $\left.1, \ldots, m_{1}\right\}$, corresponding to the columns of $\Gamma^{1}$. Theorem 9 gives conditions under which $S^{1}=\operatorname{span}\left\{\gamma_{1}, \ldots, \gamma_{m_{1}}\right\}$ and $S_{\kappa}^{1}=\operatorname{span}\left\{\Gamma^{1} v_{\kappa}^{1}(x)\right\}$ are identical subspaces (as well as $S=S_{\kappa}$ ). 
Example 5. Returning to the example of Remark 10, we have

$$
\Gamma^{1}=\left(\begin{array}{rr}
1 & -1 \\
0 & 0
\end{array}\right), \quad \Gamma^{2}=\left(\begin{array}{rr}
-2 & 1 \\
1 & -1
\end{array}\right)
$$

hence $E=\exp \left(x_{2}\right)$ is a linear exponential factor with cofactor $\omega \Gamma^{2} v_{\kappa}^{2}(x)=$ $k_{3} x_{1}-k_{4} x_{2}$ and $\omega=(0,1)$. Also

$$
\Gamma_{\kappa}^{1}=\left(\begin{array}{c}
k_{1}-k_{2} \\
0
\end{array}\right), \quad \Gamma_{\kappa}^{2}=\left(\begin{array}{rr}
-2 k_{3} & k_{4} \\
k_{3} & -k_{4}
\end{array}\right), \quad \text { for } \quad k_{1} \neq k_{2},
$$

and we reach the same conclusion as in the first case. Here $d_{\kappa}=d^{\prime}=2$. Finally,

$$
\Gamma_{\kappa}^{1}=\left(\begin{array}{cc}
-2 k_{3} & k_{4} \\
k_{3} & -k_{4}
\end{array}\right), \quad \text { for } \quad k_{1}=k_{2}
$$

with $\Gamma_{\kappa}^{2}$ empty and there are no linear exponential factors. Here $d_{\kappa}=1<d^{\prime}$.

\section{Darboux integrability and reaction network structure}

Here we explore a connection between the existence of certain Darboux polynomials and a structure of reaction networks.

Theorem 20. Let 22 be an n-dimensional ODE system for a reaction network with mass-action kinetics, as in (3). Then for $1 \leq i \leq n$ the following statements are equivalent:

(i) $F=x_{i}$ is a Darboux polynomial with cofactor $K \in \mathbb{R}[x]$.

(ii) If $\alpha_{j i}^{\prime}>0$, then $\alpha_{j i}>0$ for all $j=1, \ldots, m$.

Proof. Assume (ii). Consequently $x_{i}$ is a factor of $\left(\alpha_{j i}^{\prime}-\alpha_{j i}\right) x^{y_{j}}$, if the term is not zero. If $\alpha_{j i}^{\prime}=0$ and $\alpha_{j i}>0$, then $x_{i}$ is also a factor of $\left(\alpha_{j i}^{\prime}-\alpha_{j i}\right) x^{y_{j}}=$ ${ }_{220}-\alpha_{j i} x^{y_{j}}$ by definition of mass-action kinetics. Hence $P_{i}=\sum_{j=1}^{m}\left(\alpha_{j i}^{\prime}-\alpha_{j i}\right) x^{y_{j}}$ factorises as $P_{i}=K x_{i}$ for some polynomial $K \in \mathbb{R}[x]$. If $P_{i}$ is zero, then $K=0$ and (i) is proved. 
Conversely, assume (i), that is, $P_{i}=K x_{i}$ (which can be zero), for some $K \in \mathbb{R}[x]$, and $\alpha_{j i}^{\prime}>0$ for some $j=1, \ldots, m$. We need to show that $\alpha_{j i}>0$. Assume the opposite that $\alpha_{j i}=0$. Then by definition of mass-action kinetics $\alpha_{j i}^{\prime} k_{j} x^{y_{j}}$ is a term in $P_{i}$ not involving $x_{i}$. Hence it must cancel with terms from other reactions with the same monomial $x^{y_{j}}$, that is, $0=\sum_{\left\{j^{\prime}: y_{j^{\prime}}=y_{j}\right\}}\left(\alpha_{j^{\prime} i}^{\prime}-\right.$ $\left.\alpha_{j^{\prime} i}\right) k_{j^{\prime}} x^{y_{j}}$. Since $x_{i}$ is not a factor of $x^{y_{j}}$, we have $\alpha_{j^{\prime} i}=0$, and hence $0=$ $\sum_{\left\{j^{\prime}: y_{j^{\prime}}=y_{j}\right\}} \alpha_{j^{\prime} i}^{\prime} k_{j^{\prime}} x^{y_{j}} \geq \alpha_{j i}^{\prime} k_{j} x^{y_{j}}$, implying $\alpha_{j i}^{\prime}=0$, and we have reached a contradiction.

Condition (ii) is independent of the parameters of the reaction network, and hence it is fulfilled for all rates $\kappa$ or for none at all. Thus, we have given a structural characterisation of networks with Darboux polynomial $F=x_{i}$.

System (6) fulfils all three statements of Theorem 20 with $K=k_{1}-k_{2}$. Also system (5) fulfils all three statements of Theorem 20 for $i=1$, that is, $F=x_{1}$ is a Darboux polynomial.

Example 6 (Volpert's network, part 3). Consider Volpert's reaction network (1) with mass-action kinetics. By Theorem 16, finding

$$
\left(\begin{array}{c}
3+2-1 \\
3
\end{array}\right)+1=5
$$

Darboux polynomials guarantees the existence of a conservation law. However, fewer suffice if the linear combination of their cofactors equals to zero, see Theorem 16. The system admits the Darboux polynomials $F_{i}(x)=x_{i}$ for $i=1,2,3$ with cofactors

$$
K_{1}=k_{3} x_{3}-k_{1} x_{2}, \quad K_{2}=k_{1} x_{1}-k_{2} x_{3}, \quad K_{3}=k_{2} x_{2}-k_{3} x_{1} .
$$

Since the above cofactors are linearly dependent $k_{2} K_{1}+k_{3} K_{2}+k_{1} K_{3}=0$, the network admits, according to Theorem 16 , the Darboux first integral

$$
H_{1}=x_{1}^{k_{2}} x_{2}^{k_{3}} x_{3}^{k_{1}},
$$


for any values of the reaction rate constants $k_{1}, k_{2}, k_{3}>0$. In addition, according to Proposition 8 and Theorem 9 there is one independent linear conservation law $H_{0}=x_{1}+x_{2}+x_{3}$.

Theorem 21. Consider a reaction network with mass-action kinetics with rate constants $\kappa$. If it admits the first integral $H=\prod_{i=1}^{n} x_{i}^{\lambda_{i}} e^{\delta_{i} x_{i}}, \lambda_{i}, \delta_{i} \in \mathbb{C}$, then

(i) $\left(\delta_{1}, \ldots, \delta_{n}\right) \Gamma_{\kappa}^{1}=0$, with $\Gamma_{\kappa}^{1}$ as in Remark 19 .

(ii) if $\alpha_{j i}^{\prime}>0$ then $\alpha_{j i}>0$ for all $j=1, \ldots, m$ and $i \in I=\left\{k \mid \lambda_{k} \neq 0\right\}$.

Proof. (i) For all $x \in \mathbb{R}_{>0}^{n}, \log (H)$ is well defined. If $H$ is a first integral then so is $\log (H)=\sum_{i=1}^{n} \lambda_{i} \log \left(x_{i}\right)+\delta_{i} x_{i}$, which implies that

$$
\mathcal{D}[\log (H)]=\sum_{i \in I} P_{i} \frac{\lambda_{i}}{x_{i}}+\sum_{i=1}^{n} P_{i} \delta_{i}=0
$$

where $I=\left\{k \mid \lambda_{k} \neq 0\right\}$. Let $\delta=\left(\delta_{1}, \ldots, \delta_{n}\right)$. If $P_{i}=0$ for all $i \in I$, then $\sum_{i=1}^{n} \delta_{i} x_{i}$ is a conservation law and $\delta \Gamma_{\kappa}=0$, hence also $\delta \Gamma_{\kappa}^{1}=0$. If $P_{i} \neq 0$ for some $i \in I$ then, with $G=\sum_{i=1}^{n} \delta_{i} x_{i}$, the derivation

$$
\mathcal{D}(G)=\sum_{i=1}^{n} P_{i} \delta_{i}=-\sum_{i \in I} P_{i} \frac{\lambda_{i}}{x_{i}}
$$

has degree less than that of the system, because $x_{i}$ divides $P_{i}$ for all $i \in I$. Hence $E=e^{G}$ is an exponential linear factor and $\delta \Gamma_{\kappa}^{1}=0$ according to Remark 19 and Proposition 18

(ii) If $H$ is a first integral then $x_{i}$ is a Darboux polynomial of $P_{i}$ for $i \in I$ 7. Then (ii) follows from Theorem 20

The proof shows that under the assumptions of the theorem, there is a linear combination of cofactors for the Darboux polynomials $F_{i}=x_{i}$ and the linear exponential factors $G=e^{\sum_{i=1}^{n} \delta_{i} x_{i}} G_{i}=e^{\delta_{i} x_{i}}$, yielding the first integral $H$. The converse statement in general is not true, see Example 9

If the stoichiometric space fulfils $S^{1}=S_{\kappa}^{1}$, then $\Gamma_{\kappa}^{1}$ can be replaced by $\Gamma^{1}$. In that case $\left(\delta_{1}, \ldots, \delta_{n}\right)$ might be chosen independently of the rate constants. 
The same is not true for the constants $\left(\lambda_{1}, \ldots, \lambda_{n}\right)$.

Example 7 (Lotka-Volterra network). Consider the following version of the Lotka-Volterra system in $\mathbb{R}_{\geq 0}^{2}$,

$$
A_{1} \stackrel{k_{1}}{\longrightarrow} 2 A_{1}, \quad A_{1}+A_{2} \stackrel{k_{2}}{\longrightarrow} 2 A_{2}, \quad A_{2} \stackrel{k_{3}}{\longrightarrow} 0 .
$$

Since Theorem 20(ii) holds for this system, $F_{1}=x_{1}$ and $F_{2}=x_{2}$ are Darboux polynomials. The corresponding cofactors are

$$
K_{1}=k_{1}-k_{2} x_{2}, \quad K_{2}=k_{2} x_{1}-k_{3}
$$

Using Proposition 18 we find that $E=\exp \left(x_{1}+x_{2}\right)$ is a linear exponential factor with cofactor $L=k_{1} x_{1}-k_{3} x_{2}$. (In fact, it is the only linear exponential factor with non-zero cofactor.) Note that $k_{2} L+k_{3} K_{1}+k_{1} K_{2}=0$, hence

$$
H=x_{1}^{k_{3}} x_{2}^{k_{1}} e^{k_{2}\left(x_{1}+x_{2}\right)}, \quad\left(x_{1}, x_{2}\right) \in \mathbb{R}_{>0}^{2}
$$

is a first integral of the reaction network, which is well known [25]. By considering $H^{1 / k_{2}}$, we have $\delta_{1}=\delta_{2}=1$.

A species that is involved in its own production or degradation is said to be autocatalytic 3. Condition (ii) therefore says that the given species in the network need to be autocatalytic. The reaction network in Example 7 is perhaps a very simple example of this. The general Lotka-Volterra equation can also be made to have this property:

Example 8 (General Lotka-Volterra network). The general Lotka-Volterra equation takes the form

$$
\dot{x}_{i}=r_{i} x_{i}+\sum_{j=1}^{n} a_{i j} x_{i} x_{j}, \quad i=1, \ldots, n
$$


see [13. The equation is consistent with a reaction network by defining

$$
\begin{gathered}
A_{i} \stackrel{r_{i}}{\longrightarrow} 2 A_{i} \quad \text { for } \quad r_{i}>0, \quad \text { and } A_{i} \stackrel{\left|r_{i}\right|}{\longrightarrow} 0 \text { for } r_{i}<0, \\
A_{i}+A_{j} \stackrel{a_{i j}}{\longrightarrow} 2 A_{i}+A_{j} \quad \text { for } \quad a_{i j}>0, \quad \text { and } \quad A_{i}+A_{j} \stackrel{\left|a_{i j}\right|}{\longrightarrow} A_{j} \text { for } a_{i j}<0,
\end{gathered}
$$

where $i, j=1, \ldots, n$, and $i=j$ is allowed. If $a_{i j}=-a_{j i}$ for some $i, j$, then the two reactions might be lumped into one reaction as in Example 7.

The reaction network does not have any linear conservation laws unless $r_{i}=$ 0 for all $i=1, \ldots, n$. Since Theorem 20(ii) holds for this system, $F_{i}=x_{i}$ is Darboux polynomial for all $i=1, \ldots, n$. In the following we assume $a_{i j} \neq 0$ for at least one pair of $i, j$. We first order the bimolecular reactions and define the unique $\left(i_{k}, j_{k}\right)$ such that $a_{i_{k} j_{k}} \neq 0$ for the $k$ th reaction. By construction of the reaction network, there is at most one other reaction, say the $k^{\prime}$ th reaction, such that $\left(i_{k^{\prime}}, j_{k^{\prime}}\right)=\left(j_{k}, i_{k}\right)$. Then the matrices $\Gamma^{1}$ and $\Gamma_{\kappa}^{1}$ become

$$
\left(\Gamma^{1}\right)_{i k}=\operatorname{sign}\left(a_{i j_{k}}\right), \quad \text { and } \quad\left(\Gamma_{\kappa}^{1}\right)_{i_{k} k}=a_{i_{k} j_{k}}, \quad\left(\Gamma_{\kappa}^{1}\right)_{j_{k} k}=a_{j_{k} i_{k}} .
$$

In particular, there is only one non-zero element in each column of $\Gamma^{1}$, and at most two non-zero elements in each column of $\Gamma_{\kappa}^{1}$. In this case, for notational convenience, this column appears twice in $\Gamma_{\kappa}^{1}$, one for reaction $k$ and one for the corresponding reaction $k^{\prime}$ with indices $\left(i_{k^{\prime}}, j_{k^{\prime}}\right)=\left(j_{k}, i_{k}\right)$.

If the $i$ th species is not used in any of the bimolecular reactions, then the $i$ th unit vector $e_{i}$ fulfils $e_{i} \Gamma^{1}=e_{i} \Gamma_{\kappa}^{1}=0$. For $\Gamma^{1}$, these vectors are the only vectors fulfilling $\omega \Gamma^{1}=0$. For $\Gamma_{\kappa}^{1}$ there might be additional (reaction rate dependent) vectors fulfilling $\omega \Gamma_{\kappa}^{1}=0$. This depends on the rank of $\Gamma_{\kappa}^{1}$. If there are sufficiently many we might invoke Theorem 17 and conclude the existence of a non-trivial first integral.

Example 9 (Reversible network). Consider the following reaction network with mass-action kinetics

$$
2 A_{1}+A_{2} \stackrel{k_{1}}{\longrightarrow} A_{1}+2 A_{2}, \quad A_{1}+2 A_{2} \stackrel{k_{2}}{\longrightarrow} 2 A_{1}+A_{2} .
$$


The reaction network is said to be reversible because the second reaction is the first reversed. Although Theorem 20(ii) holds and $F_{1}=x_{1}$ and $F_{2}=$ $x_{2}$ are Darboux polynomials with cofactors $K_{1}=k_{1} x_{2}^{2}-k_{2} x_{1} x_{2}$ and $K_{2}=$ $-k_{1} x_{1} x_{2}+k_{2} x_{1}^{2}$, respectively, they are not linearly dependent and there is no first integral as in Theorem 21. Since $H=x_{1}+x_{2}$ is a linear conservation law, we can reduce the system to a one dimensional ODE, which cannot have a conservation law as in Theorem 21 (unless trajectories are constant). In this $\operatorname{case} \operatorname{rank}(\Gamma)=\operatorname{rank}\left(\Gamma^{1}\right)=1$, so Proposition 18 cannot assist further.

It follows from the so-called deficiency zero theorem that the network in Example 9 has precisely one steady state in each stoichiometric compatibility class (given by $H=x_{1}+x_{2}$ ), which is asymptotically stable. The system does not admit periodic orbits as it is one dimensional [8].

\section{Conservation laws and reaction network dynamics}

Here we show by examples how to apply the Darboux method to determine non-linear conservation laws of mass-action reaction networks. We demonstrate how first integrals can be used to study dynamic properties of the system. For that purpose we use different generalizations of Volpert's reaction network (Example 1) that fall into the class of reaction networks characterized in Theorem 20.

We start with a definition of persistence and a lemma.

Definition 22 (Persistence). An ODE system for a reaction network is said to be persistent if any trajectory starting from a positive initial point is bounded away from the boundary of $\mathbb{R}_{\geq 0}^{n}$, that is,

$$
\liminf _{t \rightarrow \infty} x_{i}(t)>0 \text { for } i=1, \ldots, n \text {. }
$$

Lemma 23. Consider a reaction network with mass-action kinetics and reaction 
rate constants $\kappa$. Assume the system admits the following first integrals

$$
H_{0}=\prod_{i=1}^{n} x_{i}^{\lambda_{i}} e^{\delta_{i} x_{i}}, \quad H_{k}=\sum_{i \in I_{k}} \omega_{i} x_{i}, \quad k=1, \ldots, r
$$

where $r \in \mathbb{N}, \omega_{i}, \lambda_{i}>0$ are positive constants, $\delta_{i} \in \mathbb{C}, \cup_{k=1}^{r} I_{k}=\{1, \ldots, n\}$, $I_{k} \cap I_{k^{\prime}}=\emptyset$ for $k \neq k^{\prime}$, and there are no other linearly independent linear first integrals. Then the system is persistent.

In addition, if $\delta_{i}=0$ for all $i$, then there exists at least one stable positive steady state $x^{*}=\left(x_{1}^{*}, \ldots, x_{n}^{*}\right)$ in each stoichiometric compatibility class (determined by $H_{k}, k=1, \ldots, r$ ), given by

$$
x_{i}^{*}=\frac{\lambda_{i} H_{k}}{\omega_{i} \sum_{j \in I_{k}} \lambda_{j}}, \quad i \in I_{k}, \quad k=1, \ldots, r .
$$

No trajectories apart from the steady state $x(t)=x^{*}, t \geq 0$, are attracted towards $x^{*}$, that is, there exists $\epsilon>0$, depending on the initial condition $x(0)$, such that $\left|x(t)-x^{*}\right|>\epsilon$ for all $t \geq 0$.

Proof. By assumption $H_{k} \geq 0$ for all $k=1, \ldots, r$. It follows that each concentration $x_{i}, i \in I_{k}$, is bounded by $0 \leq x_{i} \leq M:=\max \left\{M_{k} \mid k=1, \ldots, r\right\}$, where $M_{k}=H_{k} / \min \left\{\omega_{i} \mid i \in I_{k}\right\}$, and from $H_{0}$ that $x_{i}$ cannot get arbitrarily close to zero as this would imply that at least one other concentration, say $x_{j}$, would become arbitrarily large, contradicting that $x_{j} \leq M$ for all $j$. Hence the system is persistent.

Choose an element $i_{k} \in I_{k}$ for each $k$ and define $J_{k}=I_{r} \backslash\left\{i_{k}\right\}$. By inserting $\omega_{i_{k}} x_{i_{k}}=H_{k}-\sum_{i \in J_{k}} \omega_{i} x_{i}$ into $H_{0}$ with $\delta_{i}=0$ we obtain

$$
\begin{aligned}
H_{0} \prod_{i=1}^{n} \omega_{i}^{\lambda_{i}} & =\prod_{i=1}^{n}\left(\omega_{i} x_{i}\right)^{\lambda_{i}}=\prod_{k=1}^{r} \prod_{i \in I_{k}}\left(\omega_{i} x_{i}\right)^{\lambda_{i}} \\
& =\prod_{k=1}^{r}\left[\left(H_{k}-\sum_{i \in J_{k}} \omega_{i} x_{i}\right)^{\lambda_{i_{k}}} \prod_{i \in J_{k}}\left(\omega x_{i}\right)^{\lambda_{i}}\right]
\end{aligned}
$$

From the log sum inequality [4, it follows that (14) attains its global maxi- 
mum at $x^{*}=\left(x_{1}^{*}, \ldots, x_{n}^{*}\right)$, where

$$
x_{i}^{*}=\frac{\lambda_{i} H_{k}}{\omega_{i} \sum_{j \in I_{k}} \lambda_{j}}, \quad i \in I_{k}, \quad k=1, \ldots, r
$$

Any trajectory starting out at $x^{*}$ must remain there, hence $x^{*}$ is a positive steady state in the given stoichiometric class, say $C$. The steady state is also stable, which follows from the following argument. Let $B_{\epsilon}\left(x^{*}\right)$ be the open $L_{\delta}=\left\{x \in C \mid H_{0}\left(x^{*}\right)-H_{0}(x)<\delta\right\} \subseteq B_{\epsilon}\left(x^{*}\right)$. If this was not so then there would be $\epsilon>0$ and a sequence $\left(x_{n}, \delta_{n}\right)_{n \geq 1}$ with $x_{n} \in C \backslash B_{\epsilon}\left(x^{*}\right)$ and $H_{0}\left(x^{*}\right)-H_{0}\left(x_{n}\right)<$ $\delta_{n}$. As $C$ is compact the sequence $\left(x_{n}\right)_{n \geq 1}$ has an accumulation point, say $x^{\prime} \in C \backslash B_{\epsilon}\left(x^{*}\right)$, such that $x_{n_{j}} \rightarrow x^{\prime}$ as $n_{j} \rightarrow \infty$ for a subsequence $\left(x_{n_{j}}\right)_{j \geq 1}$. Since that if $A_{i} \in \Sigma$ and $\alpha_{i j}^{\prime}>0$ for some reaction $R_{j}$, then there is a species $A_{k} \in \Sigma$ such that $\alpha_{k j}>0$ for the same reaction. If all siphons of the network contain the species of a linear first integral, then the network is persistent. Any species $A_{i}$ for which $\alpha_{i j}^{\prime}>0$ constitutes a siphon, but $\Sigma=\left\{A_{i}\right\}$ will only contain the 330 $H_{0}$ is continuous as a function of $x$, it follows that $H_{0}\left(x_{n_{j}}\right) \rightarrow H_{0}\left(x^{\prime}\right)=H_{0}\left(x^{*}\right)$, as $\delta_{n} \rightarrow 0$ for $n \rightarrow \infty$. However, then $x^{\prime}=x^{*}$ as $x^{*}$ is unique, contradicting that $x^{\prime} \in C \backslash B_{\epsilon}\left(x^{*}\right)$. Consequently, $L_{\delta}=\left\{x \in C \mid H_{0}\left(x^{*}\right)-H_{0}(x)<\delta\right\} \subseteq B_{\epsilon}\left(x^{*}\right)$. This implies, that if $x(0) \in L_{\delta}$ then $x(t) \in L_{\delta}$ for all $t>0$, and that $x^{*}$ is stable.

For the last part, consider the right hand side of (14) as a continuous $H_{0}(x(t)) \rightarrow H_{0}\left(x^{*}\right)$ as $t \rightarrow \infty$. Since $H_{0}$ is constant along trajectories, it follows that $H_{0}(x(t)) \neq H_{0}\left(x^{*}\right)$, unless $x(t)=x^{*}$.

Remark 24. In 2] (see also 19]), a structural condition for persistence is introduced. A siphon $\Sigma \subseteq \mathcal{S}$ is a subset of the species set with the property support of a linear first integral if the concentration $x_{i}$ of $A_{i}$ is constant through time. 


\subsection{Generalized Volpert network, example A}

Consider the following chemical reaction network

$$
A_{1}+A_{2 i} \stackrel{k_{3 i-2}}{\longrightarrow} 2 A_{2 i}, \quad A_{2 i}+A_{2 i+1} \stackrel{k_{3 i-1}}{\longrightarrow} 2 A_{2 i+1}, \quad A_{1}+A_{2 i+1} \stackrel{k_{3 i}}{\longrightarrow} 2 A_{1},
$$

where $i=1, \ldots, \ell$. This is a generalization of the system in Example 1 for which $\ell=1$. This general system has $n=2 \ell+1$ species $\mathcal{S}=\left\{A_{1}, \ldots, A_{2 \ell+1}\right\}$, and $p=5 \ell+1$ complexes, $\mathcal{C}=\left\{A_{1}+A_{2}, \ldots, A_{1}+A_{2 \ell+1}, A_{2}+A_{3}, \ldots, A_{2 \ell}+\right.$ $\left.A_{2 \ell+1}, 2 A_{1}, \ldots, 2 A_{2 \ell+1}\right\}$. There are $m=3 \ell$ reactions.

Proposition 25. For any values of the reaction rate constants $k_{1}, \ldots, k_{3 \ell}>0$ the polynomial differential system corresponding to the reaction network in (15) admits the following two first integrals of Darboux type

$$
H_{0}=x_{1} \prod_{i=1}^{\ell} x_{2 i}^{k_{3 i} / k_{3 i-1}} \prod_{i=1}^{\ell} x_{2 i+1}^{k_{3 i-2} / k_{3 i-1}}, \quad H_{1}=\sum_{i=1}^{2 \ell+1} x_{i}
$$

the latter being the only linear first integral of the system. The first integral is the only possible first integral of the form $H_{0}=\prod_{i=1}^{n} x_{i}^{\lambda_{i}}$.

Proof. The differential equations associated with 15 are given by

$$
\begin{aligned}
\frac{d x_{1}}{d t} & =x_{1} \sum_{i=1}^{\ell}\left(-k_{3 i-2} x_{2 i}+k_{3 i} x_{2 i+1}\right), \\
\frac{d x_{2 i}}{d t} & =x_{2 i}\left(k_{3 i-2} x_{1}-k_{3 i-1} x_{2 i+1}\right), \\
\frac{d x_{2 i+1}}{d t} & =x_{2 i+1}\left(k_{3 i-1} x_{2 i}-k_{3 i} x_{1}\right),
\end{aligned}
$$

for $i=1, \ldots, \ell$. In total we have $2 \ell+1$ equations and $3 \ell$ reaction rate constants. 340 Since there are $2 \ell+1$ linkage classes, $2 \ell+1$ terminal linkage classes, and $s=$ $n-\operatorname{rank}(\Gamma)=1$, then $H_{1}$ is the only linear conservation law according to Theorem 9, for all values of the reaction rate constants.

It follows from Theorem 20 that the system admits at least $2 \ell+1$ Darboux polynomials, namely $F_{i}=x_{i}$, where $i=1, \ldots, 2 \ell+1$, with the following $2 \ell+1$ 
cofactors

$$
\begin{aligned}
K_{1} & =\sum_{i=1}^{\ell}-k_{3 i-2} x_{2 i}+k_{3 i} x_{2 i+1}, \\
K_{2 i} & =k_{3 i-2} x_{1}-k_{3 i-1} x_{2 i+1}, \\
K_{2 i+1} & =k_{3 i-1} x_{2 i}-k_{3 i} x_{1} .
\end{aligned}
$$

In order to find a Darboux first integral one needs to find $\lambda_{1}, \ldots, \lambda_{2 \ell+1}$ such that

$$
\begin{aligned}
0 & =\lambda_{1} K_{1}+\ldots+\lambda_{2 \ell+1} K_{2 \ell+1} \\
& =x_{1} \sum_{i=1}^{\ell}\left(\lambda_{2 i} k_{3 i-2}-\lambda_{2 i+1} k_{3 i}\right) \\
& +\sum_{i=1}^{\ell}\left(\lambda_{2 i+1} k_{3 i-1}-\lambda_{1} k_{3 i-2}\right) x_{2 i}+\sum_{i=1}^{\ell}\left(\lambda_{1} k_{3 i}-\lambda_{2 i} k_{3 i-1}\right) x_{2 i+1} .
\end{aligned}
$$

This expression is zero only if each coefficient of $x_{i}$ is zero. It follows that any solution is proportional to

$$
\lambda_{1}=1, \quad \lambda_{2 i}=\frac{k_{3 i}}{k_{3 i-1}}, \quad \lambda_{2 i+1}=\frac{k_{3 i-2}}{k_{3 i-1}}
$$

Hence, according to Theorem 17, $H_{1}=\prod_{i=1}^{n} x_{i}^{\lambda_{i}}$ is a Darboux first integral.

Oppositely, if $H_{0}=\prod_{i=1}^{n} x_{i}^{\lambda_{i}}$ is a first integral for some $\lambda_{i} \in \mathbb{C}$, then so ${ }_{345}$ is $\log \left(H_{0}\right)$. It follows that $\mathcal{D}\left(H_{0}\right)$ is a linear combination of the cofactors $K_{i}$, hence $\lambda_{i}$ must take the form stated above.

The assumption of Lemma 23 is fulfilled for the reaction network above; hence the conclusions apply. In particular the system is persistent. Volpert [25] showed that for $\ell=1(n=3)$ any trajectory starting in a positive initial point is periodic. Since all reactions have total degree 2 , there cannot be any non-trivial linear exponential factors. 


\subsection{Generalized Volpert network, example B}

Consider the following chemical reaction network

$$
A_{i}+A_{i+1} \stackrel{k_{i}}{\longrightarrow} 2 A_{i+1}, \quad A_{n}+A_{1} \stackrel{k_{n}}{\longrightarrow} 2 A_{1}
$$

where $i=1, \ldots, n-1$. This is a generalization of the system in Example 1 for which $n=3$. The general system has $n$ species, and $p=2 n$ complexes, $\mathcal{C}=\left\{A_{1}+A_{2}, \ldots, A_{n-1}+A_{n}, A_{n}+A_{1}, 2 A_{1}, \ldots, 2 A_{n}\right\}$. There are $m=n$ reactions. A modified system has been considered in [23], where each species is allowed to diffuse in and out, that is, the reactions $0 \rightarrow A_{i}$, and $A_{i} \rightarrow 0$ are included for $i=1, \ldots, n$.

Proposition 26. For any values of the reaction rate constants $\kappa$, the polynomial conservation law $H_{1}=\sum_{i=1}^{n} x_{i}$.

If $n \geq 3$ is odd, then for any values of the reaction rate constants $\kappa$, there is an additional non-linear conservation law

$$
H_{0, \text { odd }}=\prod_{i=1}^{n} x_{i}^{\lambda_{i}}, \quad \lambda_{i}=\prod_{j=1}^{(n-1) / 2} k_{[(2 j+i) \bmod n]+1}
$$

If $n \geq 4$ is even and the following constraint on the rate constants is fulfilled

$$
1=\frac{k_{n} k_{n-2} \ldots k_{2}}{k_{n-1} k_{n-3} \ldots k_{1}}
$$

then there are two non-linear conservation laws of the form $H_{0, \text { even }}=\prod_{i=1}^{n} x_{i}^{\lambda_{i}}$, one with

$$
\lambda_{1}=1, \quad \lambda_{2 i+1}=\frac{k_{2 i-1} \ldots k_{1}}{k_{2 i} \ldots k_{2}}, \quad \lambda_{2 i}=0,
$$

and

$$
\lambda_{2}=1, \quad \lambda_{2 i+2}=\frac{k_{2 i} \ldots k_{2}}{k_{2 i+1} \ldots k_{3}}, \quad \lambda_{2 i-1}=0,
$$

for $i=1, \ldots, \frac{n}{2}$.

Moreover, for general $n \geq 3$, any first integral of the form $H_{0}=\prod_{i=1}^{n} x_{i}^{\lambda_{i}}$ 
is a product $H_{0}=\left(H_{0, \text { even }}^{1}\right)^{\gamma} H_{0, \text { even }}^{2}$ where $\gamma \in \mathbb{C}, H_{0 \text {,even }}^{1}$ has $\lambda_{i}$ chosen as in (18), and $H_{0, \text { even }}^{2}$ has $\lambda_{i}$ chosen as in 19.

Proof. The polynomial differential system associated to the network 16 is

$$
\begin{aligned}
\frac{d x_{1}}{d t} & =x_{1}\left(k_{n} x_{n}-k_{1} x_{2}\right) \\
\frac{d x_{i}}{d t} & =x_{i}\left(k_{i-1} x_{i-1}-k_{i} x_{i+1}\right), \quad \text { for } \quad i=2, \ldots, n-1 \\
\frac{d x_{n}}{d t} & =x_{n}\left(k_{n-1} x_{n-1}-k_{n} x_{1}\right),
\end{aligned}
$$

It is straightforward to check that $H_{1}$ is a conservation law. Since there are $n$ linkage classes, $n$ terminal linkage classes and $s=n-\operatorname{rank}(\Gamma)=1$, by Theorem 9. the function $H_{1}$ is the only linear conservation law for all values of the reaction rate constants.

According to Theorem 20 system 20 admits the Darboux polynomials $F_{i}=$ $x_{i}, i=1, \ldots, n$, with cofactors given by $K_{1}=k_{n} x_{n}-k_{1} x_{2}, K_{i}=k_{i-1} x_{i-1}-$ $k_{i} x_{i+1}, K_{n}=k_{n-1} x_{n-1}-k_{n} x_{1}$, for $i=2, \ldots, n-1$. In order to identify a Darboux first integral one needs to find $\lambda_{1}, \ldots, \lambda_{n} \in \mathbb{C}$ such that

$$
\begin{aligned}
0 & =\lambda_{1} K_{1}+\ldots+\lambda_{n} K_{n} \\
& =\left(\lambda_{2} k_{1}-\lambda_{n} k_{n}\right) x_{1}+\sum_{i=2}^{n-1}\left(\lambda_{i+1} k_{i}-\lambda_{i-1} k_{i-1}\right) x_{i}+\left(\lambda_{1} k_{n}-\lambda_{n-1} k_{n-1}\right) x_{n} .
\end{aligned}
$$

This expression is zero only if each coefficient of $x_{i}$ is zero.

If $n \geq 3$ is odd, the equality 21 holds for

$$
\lambda_{1}=\prod_{j=1}^{(n-1) / 2} k_{2 j}, \quad \lambda_{i}=\prod_{j=1}^{(n-1) / 2} k_{[(2 j+i) \bmod n]+1}, \quad i=2, \ldots, n
$$

If $n \geq 4$ is even, the equations $\lambda_{i+1} k_{i}-\lambda_{i-1} k_{i-1}=0$ in 21 imply

$$
\lambda_{2 i+1}=\frac{k_{2 i-1}}{k_{2 i}} \lambda_{2 i-1}=\ldots=\frac{k_{2 i-1} \ldots k_{1}}{k_{2 i} \ldots k_{2}} \lambda_{1}
$$

and similarly for $\lambda_{2 i}$. Choosing $\lambda_{1}=1$ and $\lambda_{2}=0$ gives (18), and $\lambda_{1}=0$ and 
$\lambda_{2}=1$ gives 190 . for $\lambda_{2 i-1}, \lambda_{2 i}$ in terms of $\lambda_{1}, \lambda_{2}$, respectively. The equation $\lambda_{1} k_{n}-\lambda_{n-1} k_{n-1}=0$ gives, using 22,

$$
\lambda_{n-1}=\frac{k_{n}}{k_{n-1}} \lambda_{1}=\frac{k_{n-3} \ldots k_{1}}{k_{n-2} \ldots k_{2}} \lambda_{1}
$$

which implies the constraint (17), if $\lambda_{1} \neq 0$. Similarly, the equation $\lambda_{2} k_{1}-$ $\lambda_{n} k_{n}=0$ also implies the constraint (17), if $\lambda_{2} \neq 0$.

Conversely, if $H_{0}=\prod_{i=1}^{n} x_{i}^{\lambda_{i}}$ is a first integral for some $\lambda_{i} \in \mathbb{C}$, then so is $\log \left(H_{0}\right)$. It follows that $\mathcal{D}\left(H_{0}\right)$ is a linear combination of the cofactors $K_{i}$, hence $\lambda_{i}$ must take the form stated above.

Lemma 23 is applicable for $n$ odd. In order to apply Lemma23 for $n$ even, we need to choose two first integrals for which different $\lambda_{i}$ 's are zero and combine them into one first integral. Hence, the assumption of Lemma 23 is fulfilled and therefore also the conclusions hold. Since all reaction have total degree 2, then there cannot be any non-trivial linear exponential factors.

\section{Acknowledgements}

AM acknowledges the support of EPSRC project EP/K036157/1. AF is partially supported by the MINECO grants MTM2013-40998-P and MTM201677278-P and by the Universitat Jaume I grant P1-1B2015-16. CV is partially supported by FCT/Portugal through UID/MAT/04459/2013. CW is supported by the Lundbeck Foundation, Denmark, the Danish Research Council and Dr.phil. Ragna Rask-Nielsen Grundforskningsfond (administered by the Royal Danish Academy of Sciences and Letters). This work was finalised while CW was visiting Universitat Politècnica de Catalunya in Spring 2017.

\section{References}

[1] Aybar, I.K., Aybar, O.O., Fercec, B., Romanovski, V.G., Samal, S.S., Weber, A., 2015. Investigation of invariants of a chemical reaction system 
with algorithms of computer algebra. MATCH Commun. Math. Comput. Chem., 74:465-480.

[10] Ferragut, A., Mahdi, A., Valls, C., Wiuf, C., 2016. On the Darboux integrability of the Edelstein system in $\mathbb{R}^{3}$. Submitted.

[11] Ferragut, A., Valls, C., 2016. On the Darboux integrability of a cubic CRN model in $\mathbb{R}^{5}$. Chaos, Solitons and Fractals 82:131-138, 2016. 
[12] Ferragut, A., Valls, C., 2017. On the complete integrability of the Raychaudhuri differential system in $\mathbb{R}^{4}$ and of a CRNT model in $\mathbb{R}^{5}$. Qual. Theory Dyn. Syst. doi:10.1007/s12346-017-0230-7, 2017.

[13] Hofbauer, J., Sigmund, K., 1998. Evolutionary Games and Population Dynamics. Cambridge University Press.

[14] Jouanolou, J.P., 1979. Equations de Pfaff algébriques. Springer.

[15] Li, G., Rabitz, H., Tóth, J., 1994. A general analysis of exact nonlinear lumping in chemical kinetics. Chem. Eng. Sci., 49(3):343-361.

[16] Llibre, J., Mahdi, A., Valls, C., 2013. Darboux integrability of the Lü system. J. Geom. Phys., 63:118-128.

[17] Llibre, J., Valls, C., 2012. Liouvillian and analytic first integrals for the Brusselator system. J. Nonlin. Math. Phys., 19(02).

[18] Mahdi, A., Valls, C., 2011. Integrability of the Nosé-Hoover equation. J. Geom. Phys., 61(8):1348-1352.

[19] Marcondes de Freitas, M., Feliu, E., Wiuf, C., 2016. Intermediates, catalysts, persistence, and boundary steady states. J. Math. Biol., to appear.

[20] Nagy, I., Tóth, J., 2014. Quadratic first integrals of kinetic differential equations. J. Math. Chem., 52(1):93-114.

[21] Smirnov, G., 2002. Introduction to the theory of differential inclusions, volume 41 of 'Graduate Studies in Mathematics'. American Mathematical

[22] Szili, L., Tóth, J., 1993. Necessary condition of the Turing instability. Phys. Rev. E, 48(1):183-186.

[23] Togashi, Y., Kaneko, K., 2001. Transitions induced by the discreteness of molecules in a small autocatalytic system. Phys. Rev. Letters, 86(11):24592462. 
[24] Tóth, J., Hárs, V., 1986. Orthogonal transforms of the lorenz- and rö"sslerequations. Physica, 19D:135-144.

[25] Volpert, A.I., 1972. Differential equations on graphs. Mathematics of the USSR-Sbornik, 17(4):571.

[26] Zhang, X., Llibre, J., 2012. On the Darboux integrability of the polynomial differential systems. Qual. Theory Dyn. Syst., 11:129-144. 